\title{
No evidence of seasonality of birth in adult type 2 diabetes in Denmark
}

\author{
Camilla B. Jensen ${ }^{1,2}$ • Esther Zimmermann ${ }^{1} \cdot$ Michael Gamborg ${ }^{1}$. \\ Berit L. Heitmann ${ }^{3,4,5}$ • Jennifer L. Baker ${ }^{1,6}$ • Allan Vaag ${ }^{7}$. \\ Thorkild I. A. Sørensen ${ }^{1,6,8}$
}

Received: 12 March 2015 / Accepted: 19 May 2015 /Published online: 16 June 2015

(C) Springer-Verlag Berlin Heidelberg 2015

\begin{abstract}
Aims/hypothesis The season of birth might influence prenatal circumstances, which may influence the risk of developing type 2 diabetes. The aim of this study was to determine whether the diagnosis of type 2 diabetes in Denmark changed with the season of birth.

Methods This study used data from the population-based Copenhagen School Health Records Register (CSHRR) that includes schoolchildren born between 1930 and 1989. Via a personal identification number, the CSHRR was linked to the National Patient Register containing hospital discharge diagnoses since 1977 . The effect of seasonal variation in birth on
\end{abstract}

Camilla B. Jensen

camilla.bjoern.jensen@regionh.dk

1 Institute of Preventive Medicine, Bispebjerg and Frederiksberg Hospital, The Capital Region, Nordre Fasanvej 57, Hovedvejen, Entrance 5, 2000 Frederiksberg, Denmark

2 Faculty of Health and Medical Sciences, University of Copenhagen, Copenhagen, Denmark

3 Research Unit of Dietary Studies, Parker Institute, Bispebjerg and Frederiksberg Hospital, The Capital Region, Copenhagen, Denmark

4 The Boden Institute of Obesity, Nutrition, Exercise \& Eating Disorders, University of Sydney, Sydney, Australia

5 National Institute of Public Health, University of Southern Denmark, Odense, Denmark

6 Novo Nordisk Foundation Center for Basic Metabolic Research, Faculty of Health and Medical Sciences, University of Copenhagen, Copenhagen, Denmark

7 Department of Endocrinology, Diabetes and Metabolism, Rigshospitalet, Copenhagen, Denmark

8 MRC Integrative Epidemiology Unit, University of Bristol, Bristol, UK the risk of type 2 diabetes was assessed using Cox regression, with month or season of birth as the predictor. The underlying time variable was age, and follow-up started in 1977 or at age 30 years.

Results The study population consisted of 223,099 people, of whom 12,486 developed adult type 2 diabetes. Using January as the reference month, the risk of type 2 diabetes by month of birth was not statistically different for any of the 11 comparative birth months. Grouping month of birth into seasons (spring was the reference) gave essentially similar results, showing no difference in the risk of type 2 diabetes for any season. Repeating the analysis by sex, birth cohort and birthweight categories revealed no associations.

Conclusions/interpretation The risk of adult type 2 diabetes was not associated with month of birth in a large Danish population-based study. The results suggest that the causes of seasonality in birthweight are not causes of type 2 diabetes.

Keywords Birthweight · Denmark · Diabetes mellitus · Fetal development · Pregnancy · Registries · Seasons · Type 2 . Vitamin D

\author{
Abbreviations \\ CSHRR The Copenhagen School Health Records Register \\ NPR National Patient Register
}

\section{Introduction}

Studies have shown that suboptimal prenatal circumstances may lead to an increased risk of disease later in life, with type 2 diabetes being perhaps the most well-documented example of this association [1-4]. The mechanisms underlying prenatal programming of type 2 diabetes are not well understood, but it 
is possible that environmental influences during critical periods of prenatal life can programme the structure and function of organs and tissues that are essential for glucose homeostasis later in life [5].

Birthweight has been found to vary according to the season of birth, with peaks in birthweight in both autumn and summer $[6,7]$, and it has been suggested that risk of type 2 diabetes displays a similar seasonal variation depending on the month of birth [8-10]. Indeed, studies in American children [10] and in adult populations in the Netherlands [8] and Ukraine [9] have reported seasonal variations in type 2 diabetes development according to month of birth. The lowest type 2 diabetes prevalence in these studies was observed in people born in October to December, and the highest prevalence was in people born in January to April. These findings suggest that there may be shared aetiology and mechanisms behind the seasonality of birthweight and type 2 diabetes risk, in which fetal growth and eventual birthweight may or may not mediate the effects on type 2 diabetes risk.

Many factors vary with the season, including the availability and nutritional quality of foods, temperature, hours of sunshine, number of wet days, vitamin D synthesis (as a consequence of varying ultraviolet $\mathrm{B}$ radiation) and infection load. From historical famine studies in China, the Netherlands and Ukraine, we know that reduced energy intake during pregnancy is associated with a higher risk of type 2 diabetes in the offspring [11-13], and it is possible that the nutritional value of food is lower in winter than in summer. The winter hypothesis suggests that a cold temperature and fewer hours of sunshine during pregnancy signals a need for energy accrual and storage in the fetus, which will become programmed and last for life [14]. A study into vitamin D status during pregnancy and offspring risk of type 2 diabetes suggested a protective effect of vitamin $\mathrm{D}$, which might be reflected in the seasonal analysis [15]. Infections during pregnancy are suspected to alter metabolic programming and might therefore influence the risk of type 2 diabetes [16]. Another hypothesis involves postnatal factors including breast feeding [17]. It has been reported that children born in summer are breastfed for shorter periods than children born in winter [18], and the duration of breastfeeding has been reported to be inversely associated with the risk of type 2 diabetes later in life [17, 19].

The aim of the present study was to investigate whether the incidence of type 2 diabetes varied according to season of birth in a Danish cohort of men and women born between 1936 and 1983.

\section{Methods}

Study population The present study is based on a cohort from the Copenhagen School Health Records Register (CSHRR), a population-based register that includes virtually every schoolchild in Copenhagen municipality born between 1930 and 1989. The register includes 372,636 records and contains computerised information about 329,968 of these children (name, sex, date of birth, personal identification number), along with measures of height and weight. Information on birthweight was obtained from the birth year of 1936 onwards at the school entry examination when children were approximately 5-7 years of age. In general, mothers have been found to recall offspring birthweight very well $[20,21]$. The CSHRR is described in greater detail elsewhere [22].

Via the personal identification number, which was established on 2 April 1968, records in the CSHRR were linked to data from the National Patient Register (NPR) [23]. The NPR contains up to 20 hospital discharge diagnoses since 1 January 1977.

Type 2 diabetes was identified from hospital discharge diagnoses (i.e. action diagnoses, supplementary diagnoses or underlying diagnoses) in the NPR using ICD-8 (www. wolfbane.com/icd/icd8.htm) code 250 (diabetes mellitus) and ICD-10 (www.who.int/classifications/icd/en/2 codes E11 (non-insulin-dependent diabetes mellitus), E12 (malnutritionrelated diabetes mellitus), E13 (other specified diabetes mellitus), E14 (unspecified diabetes mellitus). The ICD-9 system was never introduced in Denmark; therefore, these codes were not included. Individuals registered in the NPR were included as cases at the first diagnosis of diabetes.

Type 1 and type 2 diabetes patients were indistinguishable until 1987 in the NPR, when ICD-8 code 249 (insulindependent diabetes mellitus) was introduced in Denmark. This means that ICD- 8 code 250 also includes type 1 diabetes until 1987. To reduce the number of type 1 diabetes patients included as cases, we applied an age cut-off of 30 years because the majority of individuals with type 1 diabetes are diagnosed before this age. The incidence of diabetes diagnosed after 30 years of age was used as an indicator of type 2 diabetes. Due to this age-cut-off, children born after 1983 were excluded.

Statistical analysis The effect of seasonal variation in birth on the risk of type 2 diabetes was assessed using Cox regression analysis, with month of birth as the predictor. Risk time was calculated from 1 January 1977 or 30 years of age, whichever was later. Risk time ended on the date of a diabetes diagnosis as registered in the NPR, death, emigration or loss to follow-up, or on 31 December 2013, whichever came first.

The exposure variable was modelled in various ways. First, the date of birth was modelled categorically as month of birth (January-December) and as season of birth (winter, December-February; spring, March-May; summer, JuneAugust; autumn, September-November).

The reference group was set to January in models that included the exposure variable in months, and spring in models that included the exposure variable in seasons. As a 
sensitivity analysis, we performed analyses using different reference months to ensure this would not influence the outcome of our analyses.

When appropriate, analyses were stratified by sex and birth cohort (birth years 1936-1939, 1940-1944, 1945-1949, 1950-1954, 1955-1959, 1960-1964, 1965-1969, 19701974, 1975-1979 and 1980-1983). To investigate potential effect modification, the analyses were repeated by time periods defined according to the patterns of seasonal variation in birthweight that we observed in a previous study [7].

To evaluate whether a potential association between season of birth and risk of type 2 diabetes was independent of birthweight, we adjusted the analysis by birthweight (continuous and by the categories $<2.75,2.75-3.25,3.25-3.75,3.75-$ 4.25 and $\geq 4.25$ ). We also repeated the analysis by groups of birthweight $(<3.25,3.25-3.75$ and $>3.75 \mathrm{~kg})$ to check for effect modification.

The assumption of proportional hazards was checked by stratifying the analysis by age at diagnosis in groups (30-39, $40-49,50-59,60-69$ and $70-80$ years).

Sensitivity analyses were performed using other type 2 diabetes definitions: patients $>40$ years of age, exclusion of E12-14 ICD10 codes (E12, malnutrition-related diabetes mellitus; E13, other specified diabetes mellitus; E14, unspecified diabetes mellitus), exclusion of patients ever diagnosed with type 1 diabetes, and diagnosis based on the National Diabetes Register (incident cases available from 1 January 1995) that in addition to information from the NPR includes information from the National Health Service Register (registration of chiropody and blood glucose measurements) and the Danish National Prescription Registry (purchase of oral glucose-lowering drugs) [24].

\section{Results}

Among those who had a personal identification number within the total population of 372,636 persons in the CSHRR, 262,743 were alive and living in Denmark on 1 January 1977 or when they turned 30 years of age. After excluding subjects who had missing information on birthweight or had a birthweight outside the range of $2.0-5.5 \mathrm{~kg}$, the final study population consisted of 223,099 children (Fig. 1). Within the final study population, 12,486 developed type 2 diabetes (Table 1); of the participants who never received a diagnosis of type 2 diabetes, 30,360 died, 4,745 emigrated, 148 were lost to follow-up and 175,360 were alive on 31 December 2013. Mean age at the first type 2 diabetes diagnosis was 56.3 years (SD 10.2 years). The proportion (\%) of individuals with type 2 diabetes was higher in males than in females for all birth months (Fig. 2).

Investigating the risk of type 2 diabetes by month of birth revealed no statistically significant difference in any of the

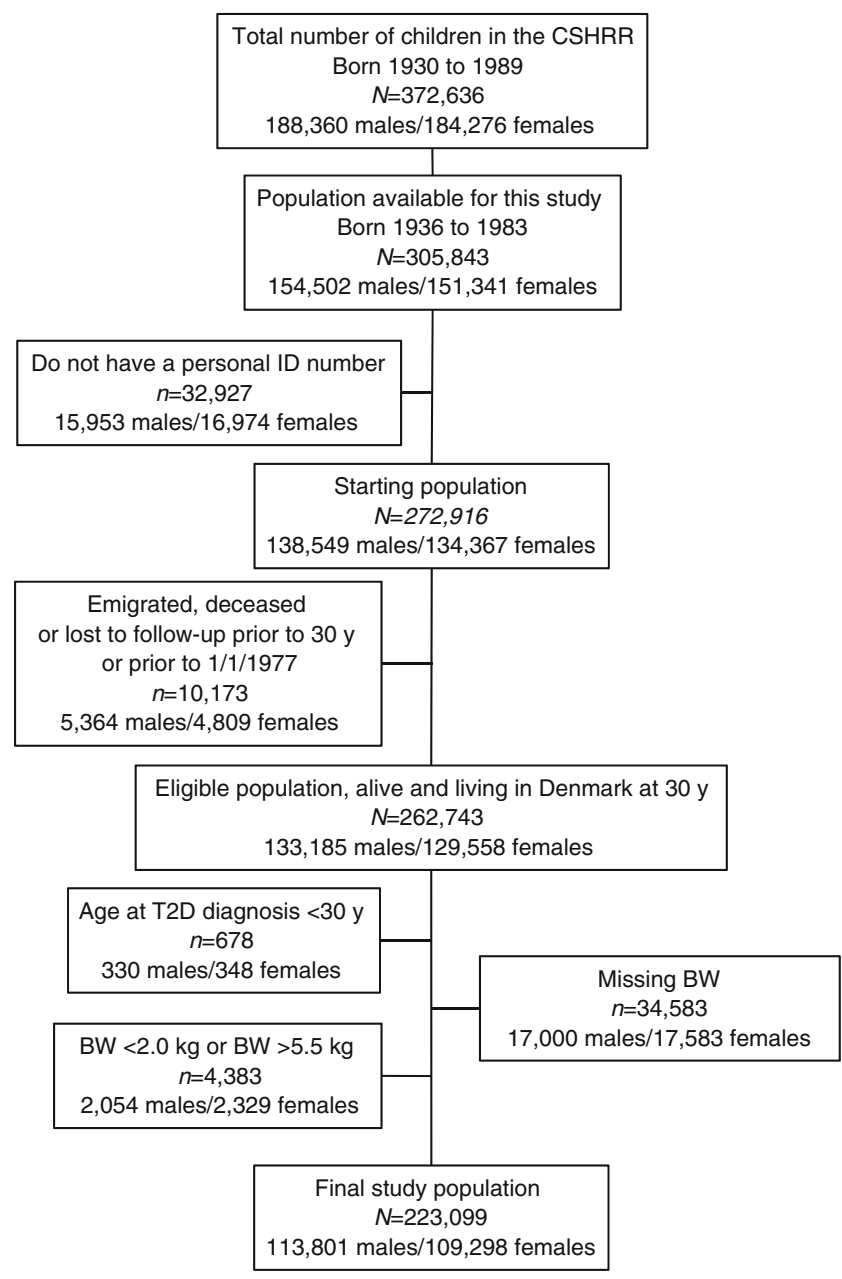

Fig. 1 Flow chart of eligible subjects and those included in the study. The study population includes children born between 1936 (when birth weight was first reported) and 1983 (due to the lower age limit for age at diagnosis of 30 years), who were alive in 1977 or at 30 years of age, and with available birthweight data. BW, birth weight; T2D, type 2 diabetes; $\mathrm{y}$, years

birth months compared with the reference month of January (Fig. 3). Changing the reference month to any other month did not change the results (data not shown). There was no notable difference in the seasonal pattern in type 2 diabetes risk between males and females (Fig. 3).

Grouping month of birth into seasons before calculating the HR of type 2 diabetes showed no association between the season of birth and risk of type 2 diabetes when comparing any other season with spring (results not shown). Changing the reference group did not influence the results (data not shown).

We wanted to see whether the hypothesised association between month of birth and later risk of type 2 diabetes was independent of birthweight. Repeating the analysis of month of birth and type 2 diabetes by birthweight categories $(<3.25$, $3.25-3.75,>3.75 \mathrm{~kg}$ ) revealed no effect modification (results not shown). When adjusting the analysis for birthweight 
Table 1 Description of type 2 diabetes cases by ICD codes

\begin{tabular}{|c|c|c|c|c|c|}
\hline \multirow[t]{2}{*}{ Classification } & \multirow[t]{2}{*}{ Code } & \multirow[t]{2}{*}{ Description } & \multicolumn{3}{|c|}{ Patients $(n)$} \\
\hline & & & Men & Women & Total \\
\hline ICD-8 & 250 & Diabetes mellitus & 962 & 551 & 1,513 \\
\hline \multirow[t]{4}{*}{ ICD-10 } & E11 & Non-insulin-dependent diabetes mellitus & 6,204 & 3,852 & 10,056 \\
\hline & E12 & Malnutrition-related diabetes mellitus & 14 & 9 & 23 \\
\hline & E13 & Other specified diabetes mellitus & 66 & 36 & 102 \\
\hline & E14 & Unspecified diabetes mellitus & 504 & 288 & 792 \\
\hline Total & & & 7,750 & 4,736 & 12,486 \\
\hline
\end{tabular}

(continuous and in categories), we saw that the risk of type 2 diabetes was significantly associated with birthweight, but the association between month of birth and risk of type 2 diabetes was unchanged (results not shown). Adjusting for birthweight in models of season of birth and risk of type 2 diabetes also showed no associations (results not shown).

We stratified the analysis by age at diagnosis and used alternative type 2 diabetes definitions, but none of the sensitivity analyses revealed an association between birth month and risk of type 2 diabetes (results not shown). Further, we found no effect modification of time periods defined according to the patterns of seasonal variation in birthweight.

\section{Discussion}

In the present study, we investigated whether the risk of type 2 diabetes was associated with the month of birth. Our hypothesis was that pregnancy during different parts of the year, and the obvious seasonal variation in various environmental exposures that follow, would influence the later risk of type 2 diabetes. However, we found no evidence to support this hypothesis in our study of Danish register-based information.

A few other studies have investigated the potential association between month of birth and risk of type 2 diabetes: in

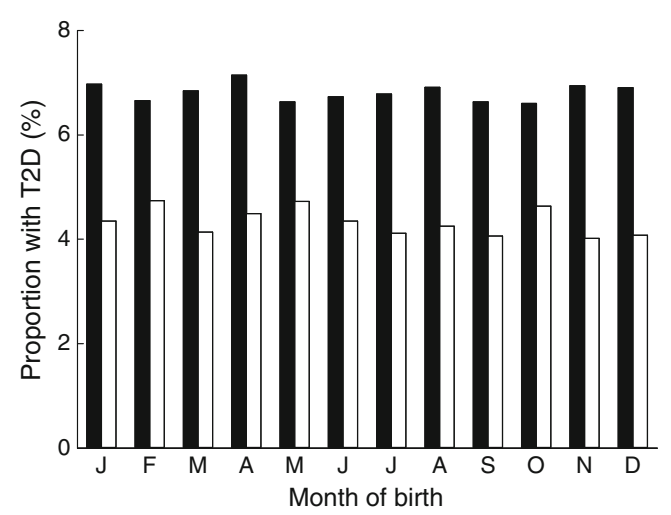

Fig. 2 The proportion of individuals with type 2 diabetes (\%) per month of birth for males (black bars) and females (white bars). T2D, type 2 diabetes contrast to our results, these studies did find significant seasonal variation in the risk of type 2 diabetes by month of birth [8-10]. We speculate that to some extent this could reflect publication bias.

In a large register-based study from the Ukraine, 52,214 type 2 diabetes patients born during the period 1920-1959, including the severe famine in Ukraine in 1933, were compared with a reference population of $1,567,917$ people born during the same period and the same region [9]. Their results showed a peak in type 2 diabetes prevalence in people born in April and a nadir in type 2 diabetes prevalence in individuals born in November-December $(p<0.0001)$. They investigated the risk in middle- to old-aged patients; the youngest patient included was 48 years at the first diagnosis. Both the time period and age group under study were similar in the Ukraine and in our study, but information on how they identified type 2 diabetes was very limited (onset after 39 years of age irrespective of type of treatment registered in the Ukrainian diabetes mellitus register). Therefore, we were unable to compare the different definitions used.

The different results observed in Ukraine and Denmark could theoretically be due to methodological differences. However, they might also be related to differences in latitude or in the standard of living between Denmark and Ukraine. Indeed, seasonal variation in both nutrition and weather was

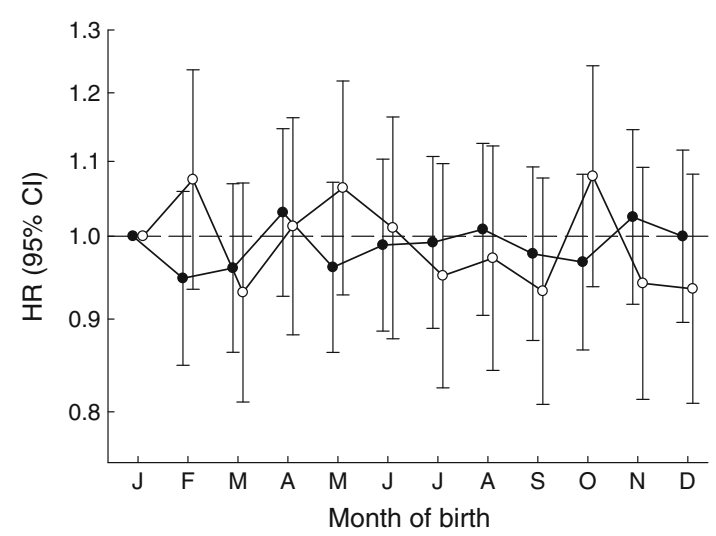

Fig. 3 HR and 95\% CIs for adult type 2 diabetes according to the month of birth in males (black circles) and females (white circles). January is the reference month 
much more extreme in Ukraine than in Denmark during the study periods; since Ukraine is a low-income country, the population might have experienced more pronounced extremes than in a more affluent country such as Denmark. Given that access to food during a famine period is likely to be most severe during spring, when food stocks are depleted, it could be speculated that the extreme famine affecting certain parts of Ukraine in 1933 may provide a partial explanation. However, the short duration of this famine period may not be sufficient to explain the seasonality of type 2 diabetes according to birth month that was seen across decades in Ukraine.

Another study that reported a seasonal variation in type 2 diabetes prevalence according to month of birth was performed in the Netherlands. It included a group of 282 type 2 diabetes patients selected from 30-90 year old individuals born during 1896-1959 in a hospital in Amsterdam, and a reference population of all births in the Netherlands during the years 1938-1970 $(n=7,591,902)$ [8]. The results showed that the risk of developing type 2 diabetes was higher in persons born during January-March compared with OctoberDecember $(p<0.005)$. Compared with the Ukraine, Denmark and the Netherlands are more similar with respect to latitude, climate and living conditions, and the study period covers some of the same years. However, one major difference is that the Dutch Hunger Winter in 1944 might have influenced the results in the Dutch study. Denmark did not experience famine during the Second World War. However, as with the Ukraine famine study, the short duration of the famine argues against this possibility.

Another study reporting that seasonal variation of birth affects type 2 diabetes risk (lowest risk of type 2 diabetes was observed in children born in October) was performed in Chicago, USA. It investigated the risk of childhood type 2 diabetes (age at diagnosis $<18$ years) according to month of birth [10]. Although type 2 diabetes was relatively prevalent among these US children, numbers were much lower than during adulthood. The childhood cases probably represent a group of more severely diseased patients with very different disease aetiologies than in adults. Also, one of the criteria they used to identify type 2 diabetes was obesity, and we did not apply this restriction to our patients. As a result, direct comparisons with our study are difficult because we did not examine the presumably severe form of type 2 diabetes that was investigated in this study. The study population was also ethnically different from ours since all participants were AfricanAmerican, and nearly all children in the CSHRR were white [22].

A common limitation of the three previous studies was the lack of information on how they identified type 2 diabetes [8-10], which is important for comparing results. Varying definitions could give groups of patients with different subtypes of the disease, which might influence the results. We identified type 2 diabetes patients in various ways, and found no differences in our results whatever definition was used. Our data were based on hospitalisation discharge diagnoses, which to an unknown extent are likely to include patients with relatively more severe type 2 diabetes. By taking this approach, and with the much higher awareness of type 2 diabetes over the last decades and the lower, more inclusive glycaemic diagnostic criteria, we probably missed an equally large number of Danish citizens with the mildest type 2 diabetes cases.

The major strengths of the present study include the large sample size, the representativeness of the cohort, and the long study period and follow-up time. Another strength is that we identified type 2 diabetes cases using the NPR, which has complete coverage of all somatic in-patients diagnosed at Danish hospitals from 1 January 1977 onwards [23]. Limitations include the late start of the NPR, which means that for the oldest participants the earliest possible diagnosis was at age 41. As mentioned above, the identification of type 2 diabetes patients at hospital admission in our study may have selected for the most severe cases. However, the sensitivity analysis identified patients based on the National Diabetes Register, which includes information from the NPR, the National Health Service Register (registration of chiropody and blood glucose measurements) and the Danish National Prescription Registry (purchase of oral glucoselowering drugs) [24]; changing the case definition had no impact on our results. The National Diabetes Register contains information on incident cases from the 1 January 1995, which means that the size of the eligible population was reduced in sensitivity analyses.

We were able to detect significant seasonal variations in birthweight in a previous study of the current Danish population [7], which support the validity of the birthweight data. This subsequently begs the question of which factors that are not fluctuating with the seasons may explain the association between low birthweight and the risk of developing type 2 diabetes. One possibility could be that genetic factors influencing both birthweight and the risk of type 2 diabetes explain the link; indeed, the MODY2 diabetes gene (also known as $G C K$ ) as well as two or three of the more than 70 known type 2 diabetes susceptibility risk genotypes are also associated with low birthweight $[25,26]$. However, these genetic associations are unlikely to explain the magnitude of the link between low birthweight and risk of type 2 diabetes, and other explanations including non-genetic factors should be looked for.

Conclusion We expected to see an association between season of birth and type 2 diabetes risk, but did not find it. The proposed pathways of association included mechanisms operating wholly or partly through birthweight, but also mechanisms that may be independent of birthweight. We found no evidence to support these hypotheses. On the other hand, we cannot exclude the possibility of mechanisms operating 
through birthweight because the amplitude of the seasonal variation in birthweight was approximately $40 \mathrm{~g}$, which may be too small to allow detection of an effect on type 2 diabetes risk. Although, in the current setting, seasonal variation in birthweight did not appear to affect the later risk of type 2 diabetes, further research in other settings with more severe seasonal variation might reveal such an association.

Funding The project was funded by the Danish Agency for Science Technology and Innovation, the Ministry of Science, Innovation and Higher Education, under the instrument 'Strategic research projects' and by a research grant from the Danish PhD School of Molecular Metabolism funded by the Novo Nordisk Foundation. A grant award from the Danish Research Counsel for Independent Research | Medical Sciences (no. 12-125974) also funded this project.

Duality of interest statement The supporting bodies for this project had no role in the design, implementation, analysis or interpretation of the data presented. The authors declare that there is no duality of interest associated with this manuscript.

Contribution statement $\mathrm{AV}$ and TIAS conceived the research idea; $\mathrm{CBJ}, \mathrm{AV}$ and TIAS designed the study; and CBJ performed the statistical analysis. All authors analysed and interpreted the data; CBJ drafted the manuscript; MG, BLH, TIAS, EZ, JLB and AV revised the manuscript critically for important intellectual content; and CBJ assumes primary responsibility for the final content. All authors read and approved the final version of the manuscript to be published. CBJ is the guarantor of this work.

\section{References}

1. Hales CN, Barker DJ, Clark PM et al (1991) Fetal and infant growth and impaired glucose tolerance at age 64. BMJ 303:1019-1022

2. Phipps K, Barker DJ, Hales CN et al (1993) Fetal growth and impaired glucose tolerance in men and women. Diabetologia 36:225-228

3. Whincup PH, Kaye SJ, Owen CG et al (2008) Birth weight and risk of type 2 diabetes: a systematic review. JAMA 300:2886-2897

4. Lithell HO, McKeigue PM, Berglund L et al (1996) Relation of size at birth to non-insulin dependent diabetes and insulin concentrations in men aged 50-60 years. BMJ 312:406-410

5. Hales CN, Barker DJ (1992) Type 2 (non-insulin-dependent) diabetes mellitus: the thrifty phenotype hypothesis. Diabetologia 35:595-601

6. McGrath J, Barnett A, Eyles D et al (2007) The impact of nonlinear exposure-risk relationships on seasonal time-series data: modelling Danish neonatal birth anthropometric data. BMC Med Res Methodol $7: 45$

7. Jensen CB, Gamborg M, Raymond K, McGrath J, Sørensen TIA, Heitmann BL (2015) Secular trends in seasonal variation in birth weight. Early Hum Dev 91:361-365
8. Jongbloet PH, van Soestbergen M, van der Veen EA (1988) Monthof-birth distribution of diabetics and ovopathy: a new aetiological view. Diabetes Res 9:51-58

9. Vaiserman AM, Khalangot MD, Carstensen B et al (2009) Seasonality of birth in adult type 2 diabetic patients in three Ukrainian regions. Diabetologia 52:2665-2667

10. Grover V, Lipton RB, Sclove SL (2004) Seasonality of month of birth among African American children with diabetes mellitus in the city of Chicago. J Pediatr Endocrinol Metab 17:289-296

11. de Rooij SR, Painter RC, Roseboom TJ et al (2006) Glucose tolerance at age 58 and the decline of glucose tolerance in comparison with age 50 in people prenatally exposed to the Dutch famine. Diabetologia 49:637-643

12. Roseboom TJ, Painter RC, van Abeelen AFM et al (2011) Hungry in the womb: what are the consequences? Lessons from the Dutch famine. Maturitas 70:141-145

13. Li Y, He Y, Qi L et al (2010) Exposure to the Chinese famine in early life and the risk of hyperglycemia and type 2 diabetes in adulthood. Diabetes 59:2400-2406

14. Foss YJ (2009) Vitamin D deficiency is the cause of common obesity. Med Hypotheses 72:314-321

15. Krishnaveni GV, Veena SR, Winder NR et al (2011) Maternal vitamin D status during pregnancy and body composition and cardiovascular risk markers in Indian children: the Mysore Parthenon Study. Am J Clin Nutr 93:628-635

16. Tamashiro KLK, Moran TH (2010) Perinatal environment and its influences on metabolic programming of offspring. Physiol Behav 100:560-566

17. Owen CG, Martin RM, Whincup PH et al (2006) Does breastfeeding influence risk of type 2 diabetes in later life? A quantitative analysis of published evidence. Am J Clin Nutr 84:1043-1054

18. Samuelsson U, Ludvigsson J (2001) Seasonal variation of birth month and breastfeeding in children with diabetes mellitus. J Pediatr Endocrinol Metab 14:43-46

19. Pettitt DJ, Forman MR, Hanson RL et al (1997) Breastfeeding and incidence of non-insulin-dependent diabetes mellitus in Pima Indians. Lancet 350:166-168

20. Adegboye ARA, Heitmann B (2008) Accuracy and correlates of maternal recall of birthweight and gestational age. BJOG 115:886-893

21. Olson JE, Shu XO, Ross JA et al (1997) Medical record validation of maternally reported birth characteristics and pregnancy-related events: a report from the Children's Cancer Group. Am J Epidemiol 145:58-67

22. Baker JL, Olsen LW, Andersen I et al (2009) Cohort profile: the Copenhagen School Health Records Register. Int J Epidemiol 38:656-662

23. Lynge E, Sandegaard JL, Rebolj M (2011) The Danish National Patient Register. Scand J Public Health 39:30-33

24. Carstensen B, Kristensen JK, Marcussen MM, Borch-Johnsen K (2011) The National Diabetes Register. Scand J Public Health 39:58-61

25. Misra S, Dornhorst A (2012) Gestational diabetes mellitus: primum non nocere. Diabetes Care 35:1811-1813

26. Garin I, Rica I, Estalella I et al (2008) Haploinsufficiency at GCK gene is not a frequent event in MODY2 patients. Clin Endocrinol (Oxf) 68:873-878 